\title{
Protopanaxtriol protects against 3-nitropropionic acid-induced oxidative stress in a rat model of Huntington's disease
}

\author{
Yan $\mathrm{GAO}^{1}$, Shi-feng $\mathrm{CHU}^{1}$, Jian-ping $\mathrm{LI}^{1}$, Zhao ZHANG ${ }^{1}$, Jia-qing $\mathrm{YAN}^{1}$, Zhi-lin WEN ${ }^{2}$, Cong-yuan XIA ${ }^{1}$, Zheng MOU ${ }^{1}$, \\ Zhen-zhen WANG ${ }^{1}$, Wen-bin $\mathrm{HE}^{1,2}$, Xiao-feng $\mathrm{GUO}^{2}$, Gui-ning WEI ${ }^{3}$, Nai-hong $\mathrm{CHEN}^{1,}$ * \\ ${ }^{1}$ State Key Laboratory of Bioactive Substances and Functions of Natural Medicines, Department of Pharmacology, Institute of Materia \\ Medica, Neuroscience Center, Chinese Academy of Medical Sciences and Peking Union Medical College, Beijing 100050, China; \\ ${ }^{2}$ Shanxi University of Traditional Chinese Medicine, Taiyuan 030024, China; ${ }^{3}$ Department of Pharmacology, Guangxi Institute of \\ Chinese Medicine \& Pharmaceutical Science, Nanning 530022, China
}

Aim: Protopanaxtriol (Ppt) is extracted from Panax ginseng Mayer. In the present study, we investigated whether Ppt could protect against 3-nitropropionic acid (3-NP)-induced oxidative stress in a rat model of Huntington's disease (HD) and explored the mechanisms of action.

Methods: Male SD rats were treated with 3-NP (20 mg/kg on d 1, and $15 \mathrm{mg} / \mathrm{kg}$ on d 2-5, ip). The rats received Ppt (5, 10, and 20 $\mathrm{mg} / \mathrm{kg}, \mathrm{po}$ ) daily prior to 3-NP administration. Nimodipine $(12 \mathrm{mg} / \mathrm{kg}, \mathrm{po})$ or N-acetyl cysteine (NAC, $100 \mathrm{mg} / \mathrm{kg}, \mathrm{po})$ was used as positive control drugs. The body weight and behavior were monitored within $5 \mathrm{~d}$. Then the animals were sacrificed, neuronal damage in striatum was estimated using Nissl staining. Hsp70 expression was detected with immunohistochemistry. Reactive oxygen species (ROS) generation was measured using dihydroethidium (DHE) staining. The levels of components in the Nrf2 pathway were measured with immunohistochemistry and Western blotting.

Results: 3-NP resulted in a marked reduction in the body weight and locomotion activity accompanied by progressive striatal dysfunction. In striatum, 3-NP caused ROS generation mainly in neurons rather than in astrocytes and induced Hsp70 expression. Administration of Ppt significantly alleviated 3-NP-induced changes of body weight and behavior, decreased ROS production and restored antioxidant enzymes activities in striatum. Moreover, Ppt directly scavenged free radicals, increased Nrf2 entering nucleus, and the expression of its downstream products heme oxygenase-1 (HO-1) and NAD(P)H quinone oxidase 1 (NQO1) in striatum. Similar effects were obtained with the positive control drugs nimodipine or NAC.

Conclusion: Ppt exerts a protective action against 3-NP-induced oxidative stress in the rat model of HD, which is associated with its anti-oxidant activity.

Keywords: protopanaxtriol; Panax ginseng; 3-nitropropionic acid; Huntington's disease; striatum; R0S; Hsp70; Nrf2 pathway; nimodipine; N-acetyl cysteine

Acta Pharmacologica Sinica (2015) 36: 311-322; doi: 10.1038/aps.2014.107; published online 2 Feb 2015

\section{Introduction}

Huntington's disease (HD) is a hereditary neurological disorder of the central nervous system (CNS) which causes progressive degeneration of striatal cells in the brain ${ }^{[1]}$. HD is caused by cytosine-adenine-guanine triplet expansion, encoding additional glutamine residues in the protein huntingtin $(\mathrm{Htt})^{[2]}$. HD patients exhibit abnormal body movements such as chorea,

\footnotetext{
* To whom correspondence should be addressed.

E-mail chennh@imm.ac.cn

Received 2014-04-18 Accepted 2014-07-28
}

cognitive impairments and personality disorders ${ }^{[3]}$. The major pathological site of HD is the striatum ${ }^{[4]}$; alterations in locomotor and motor behavior could be a result of specific effects on the striatum, which controls body movement. Mitochondrial dysfunction is a major event in the pathogenesis of HD. It has also been demonstrated that the activity of complex II of the respiratory chain is reduced in severely affected brain regions of symptomatic HD patients ${ }^{[1]}$. In the present study, we used 3-nitropropionic acid (3-NP), a toxin produced by a number of fungal and plant species, as the model agent mimicking the characteristics of $\mathrm{HD}^{[5,6]}$. 3-NP is an irreversible inhibitor 
of mitochondrial succinate dehydrogenase (SDH), which has been used to explore the molecular mechanisms of cell death associated with mitochondrial dysfunction and neurodegeneration, such as in $\mathrm{HD}^{[7,8]}$; 3-NP can significantly induce oxidative damage and impair antioxidant defense enzymes in the brain $^{[9-16]}$. It has also been reported that systemic 3-NP administration leads to oxidized proteins in the striatum, as well as a massive loss of striatal neurons ${ }^{[16]}$. These lesions may involve secondary excitotoxic oxidative stress and ATP failure mechanisms ${ }^{[11]}$.

Although HD may arise through several mechanisms, there is much evidence for the role of oxidative stress in the development of $\mathrm{HD}^{[17]}$. Reactive oxygen species (ROS) are a family of highly reactive molecules produced by stepwise, enzymatic, one-electron reductions of molecular oxygen, yielding superoxide anions and other species ${ }^{[18]}$. In the brain, the production of ROS occurs under physiological conditions, and these molecules can be neutralized by cellular antioxidant systems. However, under pathological conditions, the balance between the production and the scavenging of ROS is impaired. Oxidative stress and cellular damage result from the overproduction of ROS, ultimately causing cell death. Organisms regulate the production and scavenging of free radicals spontaneously or passively via various signaling pathways. The nuclear factor erythroid-2-related factor 2 (Nrf2) pathway, a significant signaling pathway in the detoxification of ROS in the brain region ${ }^{[19,20]}$, represents an important cellular defense mechanism against oxidative stress damage ${ }^{[21]}$. This pathway controls an array of endogenous cellular defense mechanisms against oxidative stress ${ }^{[22]}$. Under normal conditions, Nrf2 is predominantly present in the cytoplasm, bound in complex with Kelch-like ECH-associated protein1 (Keap1). When oxidative stress disrupts the Keap1-Nrf2 complex, the dissociated Nrf2 enters the nucleus, subsequently binds to the antioxidant response elements (ARE) of the phase II genes, and accelerates transcription as a defense mechanism against oxidative stress $^{[23]}$. Nrf2-regulated gene products include phase II detoxification enzymes, in addition to a range of regulators, including the enzymes $\mathrm{NAD}(\mathrm{P}) \mathrm{H}$ quinone oxidase 1 (NQO1) and heme oxygenase-1 (HO-1 $)^{[24,25]}$. NQO1 has been shown to be neuroprotective against oxidative damage by reducing highly reactive quinones to less reactive hydroquinones. HO-1 induction is one of the early events in the cell response to stress ${ }^{[26]}$.

Panax ginseng Mayer, an ancient and well-known herbal drug in traditional Chinese medicine, is a perennial herb of the family Araliaceae ${ }^{[1]}$. A large number of reports have demonstrated that panax ginseng possesses a number of beneficial effects in the CNS ${ }^{[1,27-36]}$. Protopanaxtriol (Ppt) is a neuroprotective ginseng extract. The potential neuroprotective effects of Ppt are becoming widely recognized and studied; however, the striatal anti-toxicity of Ppt is unknown. Therefore, we devised this study to verify whether Ppt can protect against 3-NP-induced oxidative stress in a rat model, to reveal the elementary mechanisms of Ppt action, and to confirm the importance of oxidative stress in the progress of neurodegenerative diseases such as HD.

\section{Materials and methods Animals}

Male Sprague-Dawley rats weighing $240 \pm 10 \mathrm{~g}$ were obtained from the Vital River Laboratory Animal Technology Co Ltd (Beijing, China, Certificate No SCXK2012-0001) and kept at a constant temperature of $23 \pm 2{ }^{\circ} \mathrm{C}$ with a $12 \mathrm{~h}$ light-dark cycle. Food and water were available ad libitum. The animals were kept in the housing facilities for one week before the experiments. All animal experiments were performed during the light phase from 9:00 to 16:00. All animal treatments were in strict accordance with the National Institutes of Health Guide for the Care and Use of Laboratory Animals.

\section{Drugs and administration}

Ppt was purchased from Jilin Province, China, and analyzed by high-performance liquid chromatography. 3-NP was purchased from Sigma-Aldrich (St Louis, MO, USA) and dissolved in saline, and the $\mathrm{pH}$ was adjusted to 7.4 with $\mathrm{NaOH}$. In this experiment, the animals received $20 \mathrm{mg} / \mathrm{kg}$ 3-NP (ip), for toxicity studies, on $\mathrm{d} 1$, and then $15 \mathrm{mg} \cdot \mathrm{kg}^{-1} \cdot \mathrm{d}^{-1}$ for the next 4 days. The rats were administered 3-NP either alone or together with $\mathrm{Ppt}$, and the control group received matching volumes of normal saline (NS). The animals were randomized and placed into one of the following 7 groups: the control group; the 3-NP $\left(80 \mathrm{mg} / \mathrm{kg}\right.$, ip) model group; the 3-NP+nimodipine $\left(12 \mathrm{mg} \cdot \mathrm{kg}^{-}\right.$ $\left.{ }^{1} \cdot \mathrm{d}^{-1}, \mathrm{po}\right)$ group; the $3-\mathrm{NP}+\mathrm{NAC}\left(100 \mathrm{mg} \cdot \mathrm{kg}^{-1} \cdot \mathrm{d}^{-1}, \mathrm{po}\right)$ group; or one of three Ppt $\left(5,10\right.$, and $\left.20 \mathrm{mg} \cdot \mathrm{kg}^{-1} \cdot \mathrm{d}^{-1}, p o\right)$ groups. The drugs or the vehicle were administered intragastrically once daily, 30 min before the 3-NP administration.

\section{Behavior test}

The behavior of the rats in all groups was immediately recorded on d 3 and graded 0 through 5: grade 0, normal behavior; grade 1 , general slowness; grade 2 , prominent gait abnormality with poor coordination; grade 3 , nearly complete hind-limb paralysis; grade 4 , inability to move; and grade 5, recumbency or death. All behavioral scoring was performed by two blinded, trained observers.

\section{Nissl staining}

Neuronal damage was estimated by staining with Nissl staining solution according to the manufacturer's instruction (Beyotime Institute of Biotechnology): the rat striatum, after paraffin embedding, were sliced to 4 - $\mu \mathrm{m}$ sections, and then paraffin sections were deparaffinized and hydrated, stained with Nissl staining solution for $30 \mathrm{~min}$ at $37^{\circ} \mathrm{C}$ and then immersed in 0.1 $\mathrm{mol} / \mathrm{L}$ phosphate buffer saline (PBS) three times for $5 \mathrm{~min}$.

\section{Immunohistochemical evaluation}

Immunohistochemical analysis was performed as follows as described: the brain sections, after dewaxing and dehydration, were incubated for antigen retrieval for $10 \mathrm{~min}$. Next, they were incubated for $10 \mathrm{~min}$ with $3 \% \mathrm{H}_{2} \mathrm{O}_{2}$ in $0.1 \mathrm{~mol} / \mathrm{L}$ PBS (pH 7.2) to block endogenous peroxidase activity and then washed 
in PBS. The sections were blocked with a solution containing $10 \%$ normal goat serum and $0.2 \%$ Triton $X-100$ for 15 min at room temperature (RT). The sections were incubated overnight at $4{ }^{\circ} \mathrm{C}$ with rabbit anti-heat shock protein 70 (Hsp70) (1:100; Cell Signaling Technology), followed by washing three times in PBS. Next, the sections were incubated with biotinylated rabbit IgG antibody (1:200; KPL, Kirkegaard \& Perry Laboratories, Inc) for $2 \mathrm{~h}$ at RT. After rinsing, the sections were incubated with biotinylated horseradish peroxidase(HRP-) complex (1:200; Kirkegaard \& Perry Laboratories, Inc) for $90 \mathrm{~min}$ at RT and visualized with 3,3'-diamino-benzidine (DAB) for $5 \mathrm{~min}$. The sections were rinsed, dehydrated, and then cover-slipped with neutral gum. The entire quantifying procedure was performed in a blinded manner.

\section{ROS detection by dihydroethidium (DHE) staining}

DHE staining was used to evaluate ROS generation. Brain sections $(4 \mu \mathrm{m})$, after dewaxing and dehydration, were incubated with DHE (100 $\mu \mathrm{mol} / \mathrm{L}$, Molecular Probes, Sigma, D7008) for $3 \mathrm{~h}$ at $37^{\circ} \mathrm{C}$, followed by three washes. Next, the sections were incubated with rabbit anti-GFAP (1:500, Dako, Glostrup, Denmark) or mouse anti-NeuN (1:200, Millipore, MAB377) antibody at $4{ }^{\circ} \mathrm{C}$ overnight. The slides were rinsed 3 times with PBS, and secondary antibodies conjugated to fluorescein were used at a dilution of $1: 200$ at $37^{\circ} \mathrm{C}$ for $1 \mathrm{~h}$. The slides were rinsed 3 times with PBS, mounted with prolong gold anti-fade mounting reagent and cover slipped. The tissue sections were then visualized with confocal laser scanning microscope, and fluorescence was detected. The images were collected and scored digitally. Image fields $(\times 40, \times 100, \times 200$, and $\times 400)$ were used to count cells displaying the specified immunofluorescent markers. The counts are expressed as the percentage of the values for the vehicle controls to normalize the differences in cell density, immunostaining, and basal survivability variations across cultures.

\section{Western blot analysis}

To investigate the levels of Nrf2, HO-1, NQO1, PCNA, and $\beta$-actin, $24 \mathrm{~h}$ after the last injection of $3-\mathrm{NP}$, the rats were anesthetized. Next, the striata were first removed with lysis buffer (50 mmol/L Tris- $\mathrm{HCl}$, pH 7.5, $150 \mathrm{mmol} / \mathrm{L} \mathrm{NaCl}$, and protease inhibitor mixture) and centrifuged at $12000 \times g$ for 30 min, following which the supernatants were collected. Protein concentrations were determined using the BCA protein assay, and equal amounts of sample were loaded on a gradient SDS-PAGE gel (10-40 $\mu \mathrm{g} /$ lane). A total of $20 \mu \mathrm{g}$ of tissue lysate from each sample was resolved. After the samples are resolved, proteins are transferred to a membrane, which is then blocked by $10 \%$ nonfat dry milk in Tween-20-containing

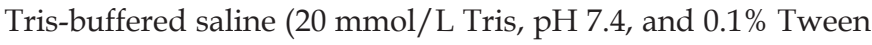
20). The membranes were probed overnight at $4^{\circ} \mathrm{C}$ with Nrf2 (C-20) antibody (1:1000, Santa Cruz Biotechnology), HO-1 antibody (1:1000, Santa Cruz Biotechnology), or NQO1 antibody (1:1000, Santa Cruz Biotechnology). This step was followed by incubation with an HRP-conjugated secondary antibody at RT for $2 \mathrm{~h}$ and exposure to charge-coupled device. For normaliza- tion of the antibody signal, the membranes were stripped and reprobed with PCNA antibody (1:1000, Santa Cruz Biotechnology) and $\beta$-actin antibody (1:2000, Santa Cruz Biotechnology). After Western blotting had been performed several times, the density of each band was converted to a numerical value using the Photoshop CS4 program (Adobe, USA), subtracting the background values from an area of film immediately adjacent to the stained band. The data are expressed as the ratios, and are normalized to the amount of $\beta$-actin.

\section{Standard enzymatic analysis with kits}

Striatum tissues at 10\% (w/v) were homogenized with NS and prepared in $0.1 \mathrm{~mol} / \mathrm{L}$ PBS ( $\mathrm{pH} 7.4$ ), followed by centrifugation at $10000 \times \mathrm{g}$ at $4^{\circ} \mathrm{C}$ for $30 \mathrm{~min}$. Next, the supernatants were separated, analyzed and used for biochemical estimations. The reaction products were determined by standard enzymatic analyses. The test kits were purchased from JianCheng Bioengineering Institute (Nanjing, China), including kits for detecting superoxide dismutase (SOD), superoxide anions, hydroxyl radicals, and SDH.

\section{Image analysis}

The number of Nissl bodies and staining for Hsp70, GFAP, NeuN, and ROS were assessed in full microscopic fields $(\times 100$, $\times 200$, and $\times 400$ ) of the striatum by using the digital medical image analysis system Image-Pro Plus software (Media Cybernetics, Silver Spring, MA, USA). Three tissue sections per animal were counted for each index.

\section{Statistical analysis}

All experiments were repeated at least three times, unless otherwise stated. The results are expressed as the mean \pm SEM. Statistical analysis of raw data was performed with Graph Pad Prism 5.0 and SPSS 17.0. The experimental groups were compared by one way analysis of variance (ANOVA), followed by Newman-Keuls test or Student's $t$-test, as appropriate. The level of statistical significance was set at $P<0.05$.

\section{Results}

\section{Ppt possessed antioxidative activity}

Ppt is the metabolic product of ginsenoside Rg1 and might have antioxidative activity (Figure 1A). First, we examined the inherent antioxidative activity of Ppt by Western blot analysis and by using antioxidant kits. We found that Ppt had an antioxidant effect in physiological conditions by analyzing the SDH activity and the levels of hydroxyl radicals and superoxide anions, as shown in Figure 1B-1D (5 and $10 \mathrm{mg} / \mathrm{kg}$ Ppt: $P<0.05)$. To further evaluate the antioxidative activity of Ppt, the classic Nrf2 pathway was examined by Western blot analysis. After Ppt administration, Nrf2 entered the nucleus, as shown in Figure 1E (5 mg/kg: $P<0.01 ; 10$, and $20 \mathrm{mg} / \mathrm{kg}$ : $P<0.05)$. Both HO-1 and NQO1, which possess antioxidative activity, were moderately expressed in the Ppt-treated groups (Figure $1 F$ and 1G). These results indicated that Ppt had an antioxidative effect in physiological conditions. 


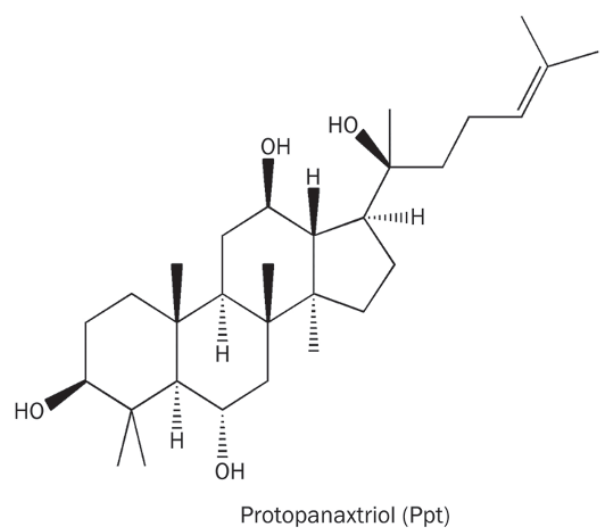

B

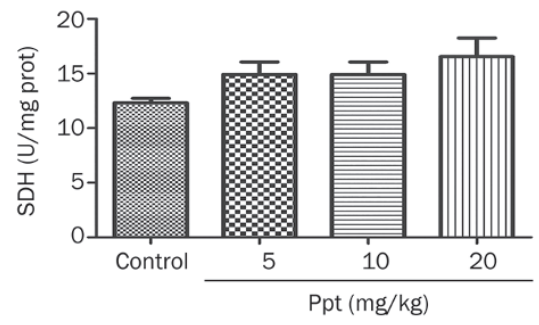

E
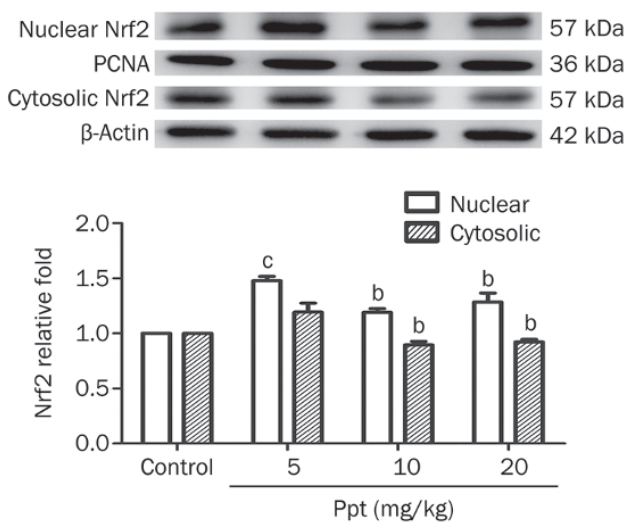

C

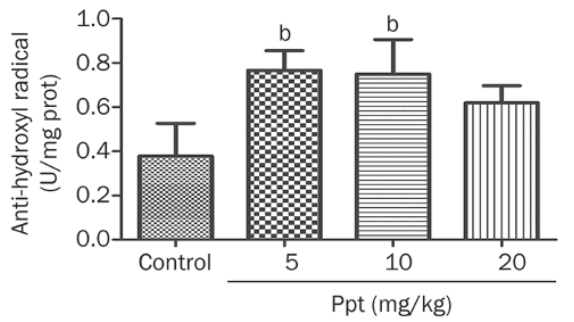

F

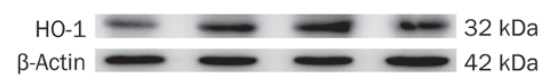

D

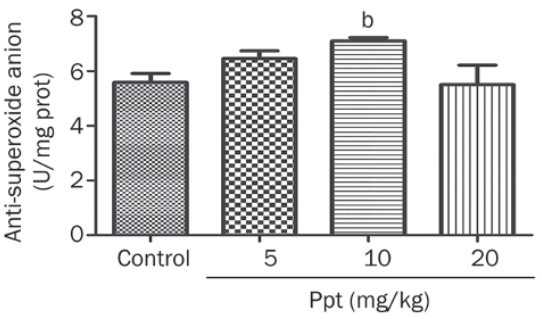

G

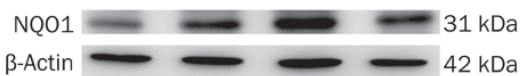

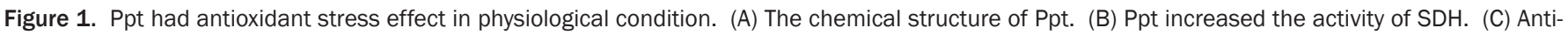

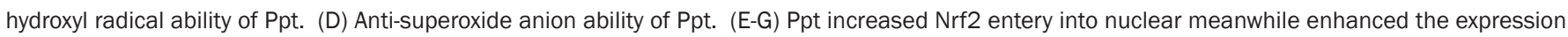
of HO-1 and NQ01. Data are expressed as the mean \pm SEM per experimental condition. ${ }^{b} P<0.05,{ }^{c} P<0.01$ vs control group.

Ppt alleviated body weight loss and ameliorated behavior disorder

3-NP treatment caused a significant decrease in body weight, compared with the control group; however, the Ppt treatment alleviated the body weight loss in the 3-NP-treated rats compared with the 3-NP alone model group $(5 \mathrm{mg} / \mathrm{kg}: P<0.01$; 10, and $20 \mathrm{mg} / \mathrm{kg}: P<0.05)$, as shown in Figure $2 \mathrm{~A}$. As shown in Figure $2 \mathrm{~B}$, the behavior in all groups was recorded on $\mathrm{d}$ 3. The behavior scores for the locomotor activity of each rat were relatively stable and showed no significant variation between groups on d 1. Intraperitoneally 3-NP treatment significantly decreased the locomotor activity on d 3 compared with the control group. In contrast, on d $3, \mathrm{Ppt}(5,10 \mathrm{mg} / \mathrm{kg}$ and nimodipine: $P<0.05 ; 20 \mathrm{mg} / \mathrm{kg}: P<0.01)$ treatment ame- liorated behavior disorders, compared with the 3-NP treated rats. From our daily observations, the first sign of striatal dysfunction was an uncoordinated and wobbling gait (d 3 of intoxication); later, the rats had transient or permanent dystonia ( $\mathrm{d} 4$ ), and by $\mathrm{d} 5$, they showed permanent dystonia of the hind limbs. The symptoms became aggravated toward recumbency, with the rats being unable to stay on their feet and lying on their sides.

\section{Ppt restored ROS impairment and protected neurons}

On the basis of the above results, we examined the location of the ROS induced by 3-NP using immunofluorescence analysis. As shown in Figures 3 and 4, 3-NP induced ROS chiefly in neurons rather than in astrocytes in the striatal regions. These 

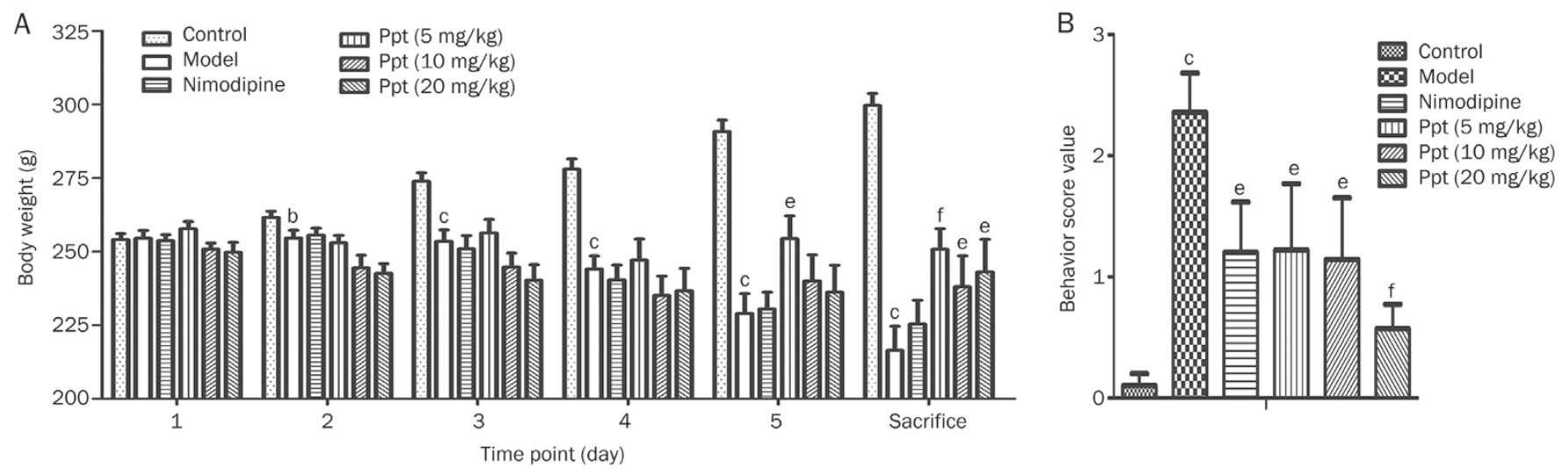

Figure 2. Ppt alleviated body weight loss and ameliorated behavior disorder. (A) Ppt alleviated body weight loss in 3-NP treated rats. (B) Ppt significantly decreased locomotor activity on d 3. Data are expressed as the mean \pm SEM per experimental condition. ${ }^{\mathrm{b}} P<0.05$, ${ }^{\mathrm{c}} P<0.01 \mathrm{vs}$ control group. ${ }^{\mathrm{e}} P<0.05,{ }^{\mathrm{f}} \mathrm{P}<0.01$ vs model group.

results also suggested that $\mathrm{Ppt}(5,10$, and $20 \mathrm{mg} / \mathrm{kg})$ treatment alleviated the overproduction of ROS in neurons, compared with the 3-NP alone model group.

3-NP induced robust production of ROS in the rat striatum, which was reduced by treatment with Ppt $(5,10$, and 20 $\mathrm{mg} / \mathrm{kg}$ ) (Figure $5 \mathrm{~A}$ ), similar to the results shown in Figures 3 and 4 . Moreover, the model group showed marked expression of Hsp70 in the striatum. However, the Ppt $(5,10$, and 20 $\mathrm{mg} / \mathrm{kg}$ ) treatment decreased this overexpression (Figure 5B). In the 3-NP group, the neurons were markedly shrunken and pyknotic, the number of cells was decreased, and some cells also displayed an irregular shape and a tangled appearance. In contrast, in the Ppt-treated groups, the nucleus showed normal morphological features, and the number of cells in the striatum decreased only sporadically, as shown using Nissl staining (Figure 5C).

\section{Ppt improved mitochondrial function and enhanced the activity of enzymes}

Mitochondria provide energy for activities required for survival; however, free radicals are also generated in this process $^{[27]}$. More than $95 \%$ of free radicals are derived from the mitochondrial respiratory chain ${ }^{[28]}$. SDH is a key enzyme in the mitochondrial respiratory chain, and SOD can protect cells against damage induced by free radicals. In the present study, systemic 3-NP administration significantly impaired mitochondrial enzyme complex II compared with the control rats. Ppt $(5,10$, and $20 \mathrm{mg} / \mathrm{kg})$ treatment significantly restored mitochondrial SDH activity compared with the 3-NP treated animals $(5$ and $10 \mathrm{mg} / \mathrm{kg}: P<0.05 ; 20 \mathrm{mg} / \mathrm{kg}: P<0.01$; Figure 6A). Furthermore, Ppt treatment increased the activity of different types of SOD, defending cells against the hypoxia induced by 3-NP (total SOD of NAC: $P<0.05$; Figure $6 \mathrm{~B}$ ). In fact, Ppt itself directly scavenged oxygen free radicals such as hydroxyl radicals and superoxide anions $(10$ and $20 \mathrm{mg} / \mathrm{kg}$ and NAC: $P<0.05 ; 5 \mathrm{mg} / \mathrm{kg}: P<0.01$, Figure $6 \mathrm{C} ; 5 \mathrm{mg} / \mathrm{kg}$ : $P<0.05 ; 10 \mathrm{mg} / \mathrm{kg}: P<0.01$; Figure $6 \mathrm{D})$, and it had a stronger ability to scavenge the hydroxyl radical than superoxide anion radicals. These results were consistent with the data above.

\section{Ppt activated the anti-oxidative stress pathway}

Finally, we examined the classic Nrf2 pathway by Western blot analysis. As shown in Figure 7A, 3-NP-induced ROS caused Nrf2 entering the nucleus, serving as a chemical sensor $^{[29]}$ to protect against oxidative stress. Nrf2 also entered the nucleus in the Ppt-treated groups, as shown in Figure 7A (5, and $10 \mathrm{mg} / \mathrm{kg}: P<0.05)$. Next, we examined the protein levels of HO-1 and NQO1 in each group. Western blot analysis revealed that both HO-1 and NQO1, which have antioxidative activity, were moderately expressed in the Ppt- and nimodipine-treated groups $(5$ and $20 \mathrm{mg} / \mathrm{kg}: P<0.05 ; 10 \mathrm{mg} / \mathrm{kg}$ and nimodipine: $P<0.01$, Figure 7B; $20 \mathrm{mg} / \mathrm{kg}: P<0.05$, nimodipine: $P<0.01$, Figure 7C). These results indicated that $\mathrm{Ppt}$ had an antioxidative effect in pathological conditions.

\section{Discussion}

The present study highlights the therapeutic potential of Ppt against 3-NP-induced oxidative damage. Our results demonstrated the following: (1) Ppt has an antioxidative effect in physiological conditions; (2) Ppt alleviates 3-NP-induced behavior disorders by increasing body weight and locomotor activity; (3) Ppt reduces the overproduction of ROS and mitigates the robust expression of Hsp70; (4) Ppt restores mitochondrial complex enzyme II and SOD activity and directly scavenges superoxide anions and hydroxyl radicals; and (5) Ppt increases Nrf2 entry to the nucleus and enhances the expression of HO-1 and NQO1. We used three dosages of Ppt $(5,10$, and $20 \mathrm{mg} / \mathrm{kg})$ for this study, and we inferred that the $10 \mathrm{mg} / \mathrm{kg}$ dosage of Ppt possesses maximum activity against 3-NP-induced experimental Huntington's disease.

3-NP could induce oxidative stress via at least three mechanisms: increasing oxygen flux by the electron transport chain, promoting excitotoxicity, and enhancing inflammatory responses to neuronal degeneration ${ }^{[30]}$. 3-NP is an irreversible inhibitor of complex II of the electron transport chain and of the Kreb's cycle. The major mechanism of 3-NP toxicity is 

NeuN
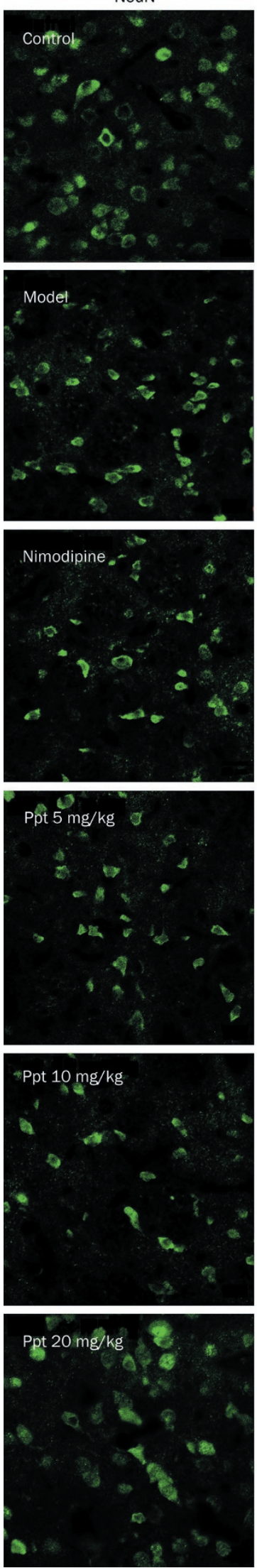

ROS
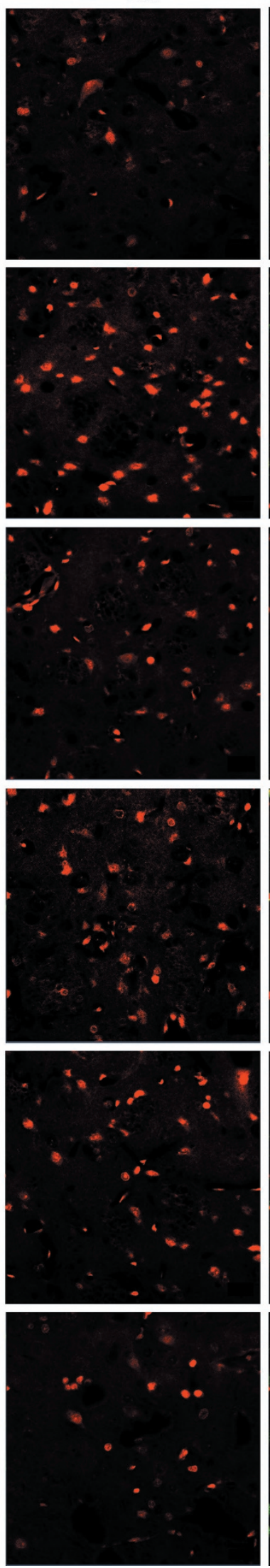

Merge
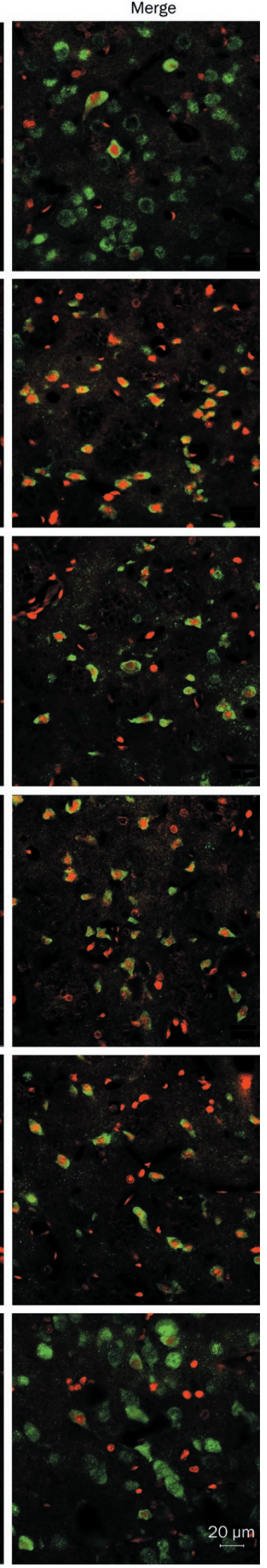

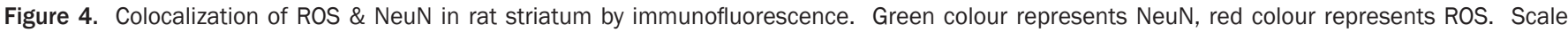
bar $=20 \mu \mathrm{m}, n=3$. 

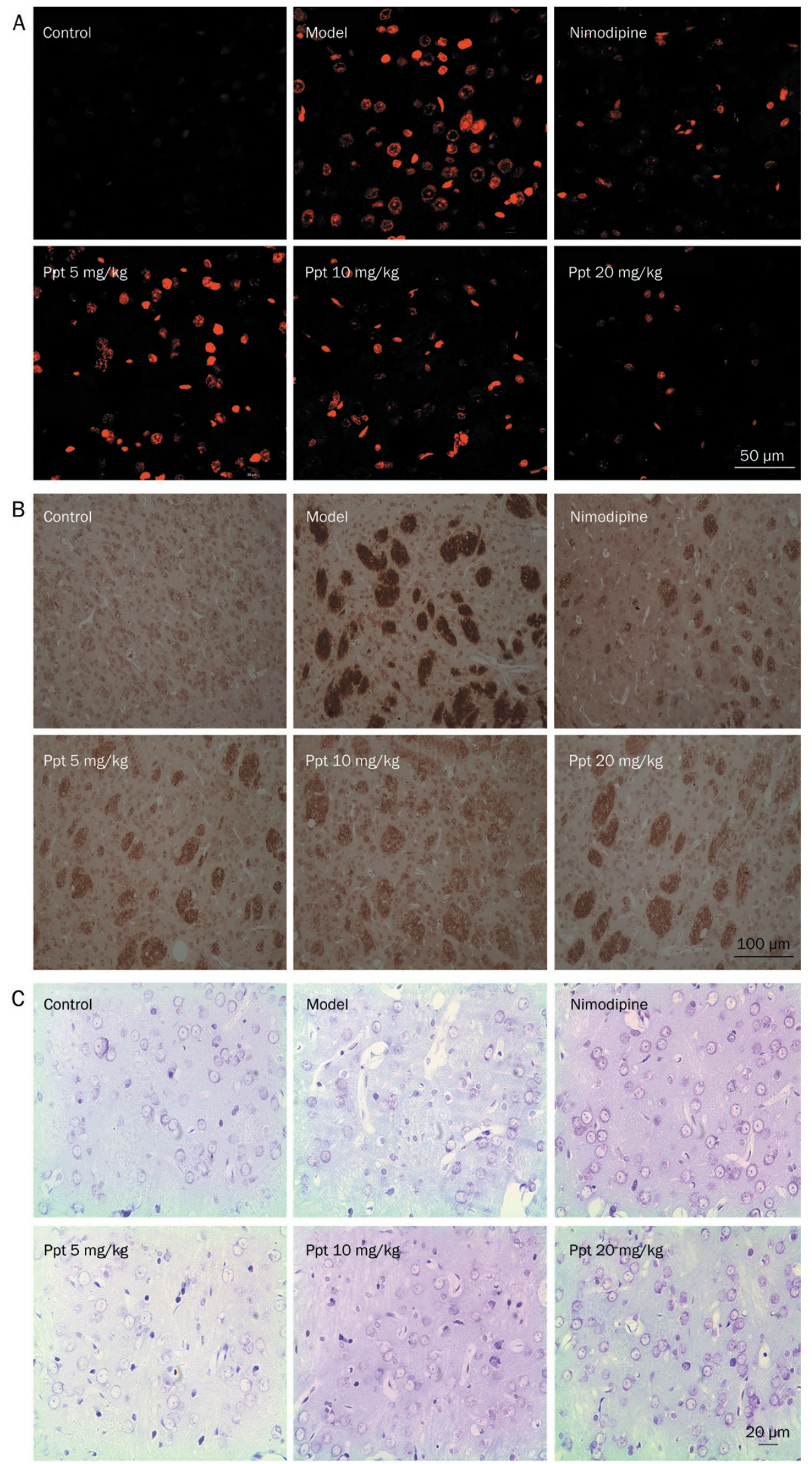

Figure 5. Ppt restored ROS impairment and protected neurons. (A) 3-NP induced robust production of ROS was reduced by Ppt in rat striatum. Scale bar $=50 \mu \mathrm{m}, n=3$. (B) Expression of Hsp70 by immunostaining of rat striatum, $\times 40$. Scale bar=100 $\mu \mathrm{m}, n=3$. (C) Niss $\mathrm{staining}$ of rat striatum neurons, $\times 200$. Scale bar $=20 \mu \mathrm{m}, n=3$. 
A

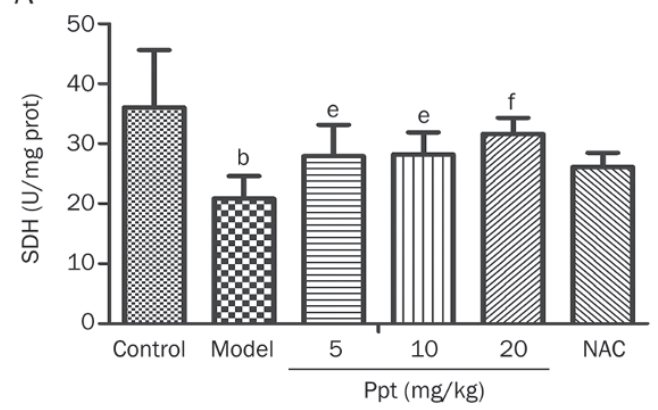

C

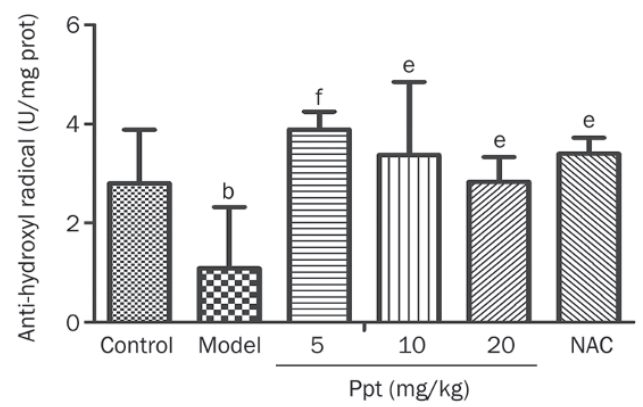

B

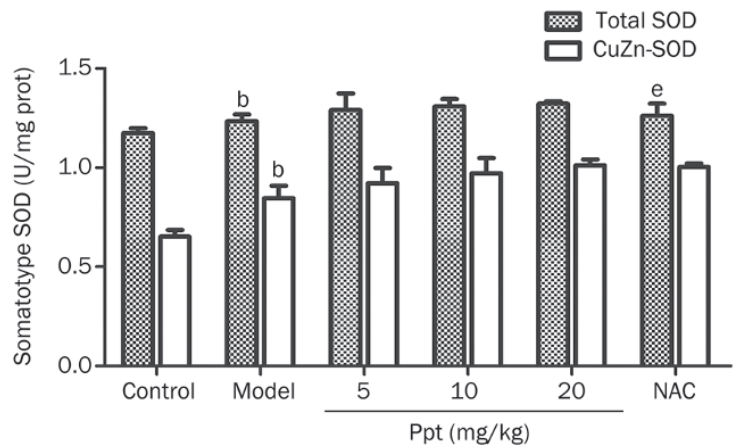

D

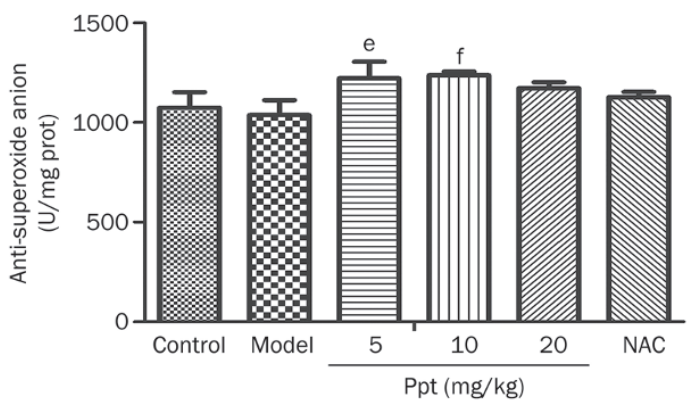

Figure 6. Ppt improved mitochondrial function and enhanced the activity of enzymes. (A) Ppt increased the activity of SDH. (B) Ppt increased the activity of somatotype SOD. (C) Anti-hydroxyl radical ability of Ppt. (D) Anti-superoxide anion ability of Ppt. Data are expressed as the mean \pm SEM per experimental condition. ${ }^{b} P<0.05$ vs control group. ${ }^{e} P<0.05,{ }^{f} P<0.01$ vs model group.

thought to be via inhibition of the citric acid cycle ${ }^{[6]}$. Oxidative stress occurs following 3-NP administration and is more widespread than the morphologic lesions ${ }^{[30]}$. In our study, intraperitoneal injection of 3-NP for 5 days caused body weight loss, motor abnormalities, and even death, suggesting that animal behavior in this model mimics HD symptoms. Intriguingly, systemic treatment with Ppt for 5 days restored the weight loss and mitigated the behavior disorders in the 3-NP treated rats. Kumar et al have shown that antioxidative treatments restore behavioral disorders and antioxidative defense mechanisms in 3-NP treated animals ${ }^{[10,11,31]}$.

In the CNS, cells are able to defend against oxidative stress using several resources, including vitamins, bioactive molecules, lipoic acid, enzymes (such as SOD, GSH, GPx, HO-1, and NQO1), and redox sensitive protein transcription factors (such as AP-1, Nrf2, and HSF) $^{[32,33]}$. Hsp70, also referred to as Hsp72, which has a cytoprotective function, is induced in many neurodegenerative disorders ${ }^{[26]}$. According to our data, Ppt inhibited the overexpression of ROS which is on behalf of oxidative stress, then the overproduction of HSP70 were reduced. Our results showed that Ppt can reduce ROS production in several ways, including the direct scavenging of the free radicals. When both Ppt and 3-NP were administered to rats, the expression of Hsp70 decreased, compared to that in the 3-NP treatment alone, suggesting that Ppt is sufficient to protect against ROS damage.

Mitochondrial respiratory chain complexes that produce ATP for cellular functioning can be damaged by 3-NP-induced neurotoxicity both in vivo and in vitro ${ }^{[11]}$. NAC is a classic antioxidant, and it has been reported that NAC increases complex I, II, and IV activities, both in vitro and in vivo in synaptic mitochondrion preparations from aged mice ${ }^{[34]}$. In fact, NAC not only decreases oxidative damage but can also increase ATP availability: it decreases ROS production by directly scavenging ROS or by promoting the synthesis of antioxidant enzymes, and it increases ATP availability by inhibiting apoptosis or influencing oxidative phosphorylation ${ }^{[34]}$. NAC treatment has been found to be effective at reversing 3-NP-induced mitochondrial dysfunction and neurobehavioral deficits ${ }^{[35]}$. Therefore, in this study, we used NAC as the positive control. 3-NP inhibited SDH activity, induced oxidative damage, and interrupted mitochondrial electron transport, causing a cellular energy deficit in the striatum ${ }^{[8]}$. Herein, 3-NP significantly altered the activities of mitochondrial complex enzymes in the striatum, and the functional activities of these enzymes were restored by Ppt treatment, which acted as an antioxidant, enhancing the activity of both SDH and SOD. CuZn-SOD is a major antioxidant enzyme that is primarily located in the cytoplasm, and Mn-SOD is a mitochondrial antioxidant enzyme essential for cell development and function ${ }^{[35]}$. Ppt prevented the CuZn-SOD enzyme inhibition induced by 3-NP. In addition, our data demonstrated that Ppt can directly scavenge ROS, including superoxide anions and, in particular, hydroxyl radicals.

Ppt exerts its antioxidative activity via several mechanisms, such as direct scavenging of free radicals and restoration of $\mathrm{SDH}$ activity. These mechanisms may contribute to its potent antioxidative and putative neuroprotective activities. Nrf2- 
A
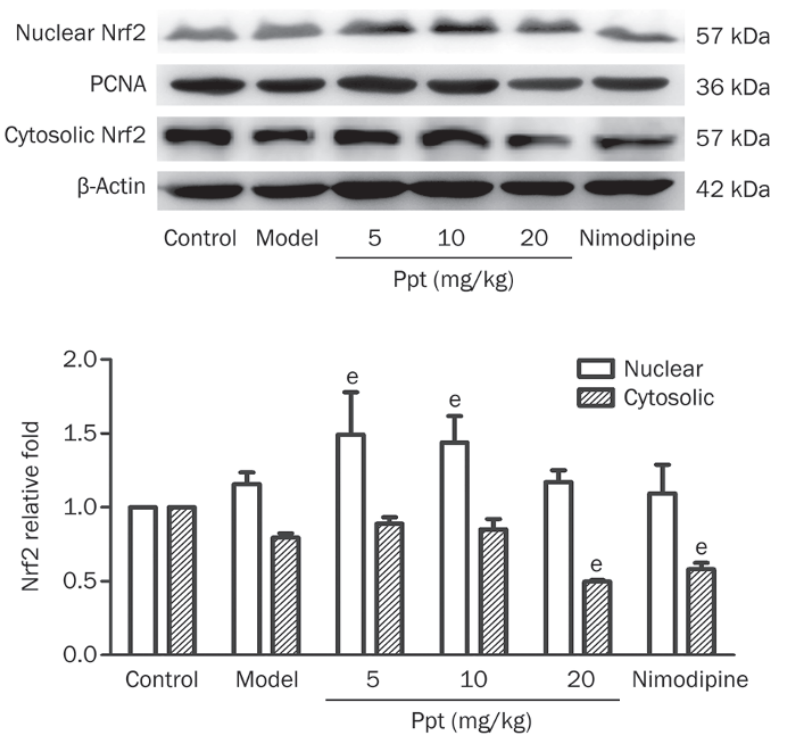

B
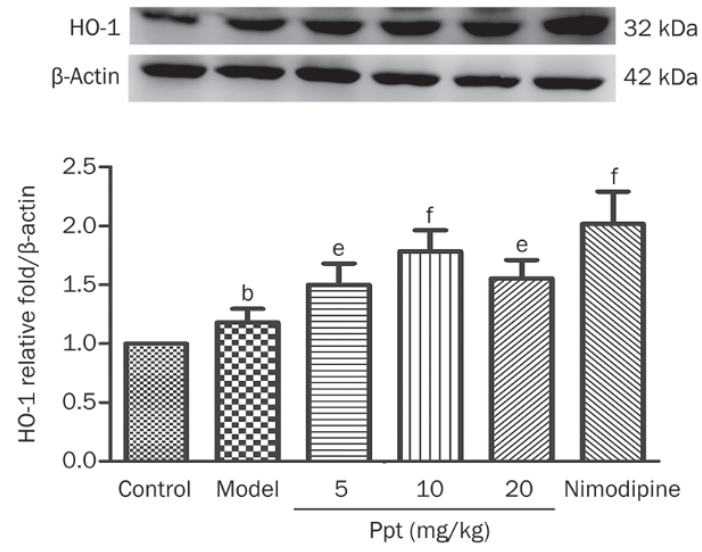

C
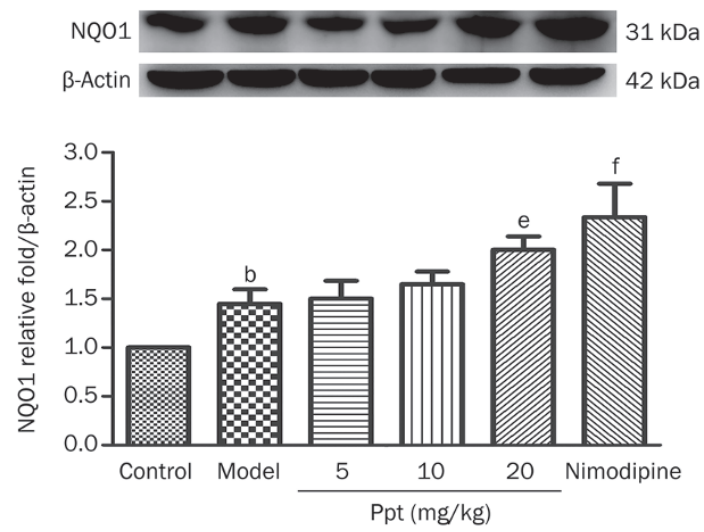

Figure 7. Ppt had antioxidant effect in pathological condition. (A-C) Ppt increased Nrf2 entery into nuclear meanwhile enhanced the expression of HO-1 and NQ01. Data are expressed as the mean \pm SEM per experimental condition. Statistical significance: ${ }^{b} P<0.05$ vs control group. ${ }^{\mathrm{e}} P<0.05$, ${ }^{\mathrm{f}} P<0.01$ vs model group.

dependent transcriptional activation by means of ARE is known to coordinate the up-regulation of cytoprotective genes involved in defending against $\operatorname{ROS}^{[36]}$; therefore Nrf2 plays an important role in reducing complex II-induced toxicity. Calkins et al demonstrated that Nrf2 is an essential inducible factor for protection against complex II inhibitor-mediated neurotoxicity and that Nrf2-mediated ARE transcription is a potential strategy for preventative therapy in neurodegenerative disorders such as $\mathrm{HD}^{[36]}$. High expression of HO-1 and NQO1 are also important for protecting neurons, and both of these enzymes are involved in downstream cascade reactions. In the present study, ROS triggered the up-regulation of the entire Nrf2 pathway and its downstream products. Ppt treatment regulated Nrf2 entry to the nucleus, enhancing the expression of HO-1 and NQO1, both in physiological and pathological conditions. There are several explanations for this phenomenon: Ppt can directly protect the function of mitochondria, inhibit the oxidative stress reaction, activate the anti-oxidative enzymes, and directly scavenge superoxide anions and especially hydroxyl radicals. We observed that the Nrf2 pathway was altered when rats were treated with both 3-NP and Ppt, suggesting that prior to the entry of a large amount of Nrf2 to the nucleus, Ppt had already scavenged ROS. Additionally, Ppt promotes Nrf2 entry to the nucleus, increasing the expression of both HO-1 and NQO1.

Astrocytes are the chief defense against ROS in the CNS. It has been reported that activated astrocytes are closely associated with neuronal death as a result of complex II inhibition and that astrocytes mount a response that may involve the activation of Nrf2 and ARE-dependent signaling ${ }^{[36]}$. The neuroprotective effect of $\mathrm{Nrf2}$ overexpression might not require Nrf2 delivery to neurons. In addition, Nrf2 expression in astrocytes appears to be a viable strategy for protecting neighboring neurons ${ }^{[37]}$. However, it is still unknown whether the Nrf2 pathway is activated primarily in neurons or in astrocytes. Moreover, if the Nrf2 pathway plays an important role in astrocytes, it is unclear how the astrocytes rescue the neurons via this pathway in the 3-NP-induced HD model. Therefore, in future studies, we will focus on the relationship between astrocytes and neurons in the 3-NP-induced rat model.

Antioxidative drugs ${ }^{[20]}$ and the overexpression of genes involved in attenuating oxidative stress showed significant neuroprotective effects against 3-NP-mediated neurotoxicity ${ }^{[11,31]}$. Ppt warrants attention as a potential anti-oxidative therapy for HD. In summary, Ppt protected against 3-NPinduced damage in the striatum, effectively decreased the levels proportion of ROS, relieved the overexpression of Hsp70, restored the morphology of neurons, and directly scavenged superoxide and especially hydroxyl radicals, reverting the organism back to the steady state. Moreover, Ppt has fewer side-effects, possesses nootropic effects and has similar or greater protective effects than the positive controls nimodipine and NAC.

\section{Conclusion}

The present study highlights the therapeutic potential of Ppt against 3-NP-induced oxidative stress in an HD model. Our 
results showed that Ppt mitigated 3-NP-induced histological and behavioral alteration (improved body weight and locomotor activity). Ppt primarily acted by promptly restoring the mitochondrial complex enzyme activity (relieving the inhibition of complex II) and by increasing the activity of antioxidative stress enzymes, as well as by directly scavenging superoxide and hydroxyl radical (chiefly hydroxyl radicals), suggesting that Ppt protects against ROS-induced neuronal damage by acting as an antioxidant. Future neurological studies involving Ppt should include therapeutic studies in both transgenic animals and human models of HD.

\section{Acknowledgements}

This work was supported by the National Natural Science Foundation of China (No 81274122, 81102831, 81173578, 81202507,81273629 , and 81373510), the National Key SciTech Major Special Item (No 2012ZX09301002-004 and 2012ZX09103101-006), the National High-Tech R\&D Programme (863 Program, No 2012AA020303), the Program for Changjiang Scholars and Innovative Research Teams in Universities (PCSIRT) (No IRT1007), the Specialized Research Fund for the Doctoral Program of Higher Education of China (№ 20121106130001), the Beijing Natural Science Foundation (No 7131013 and 7142115), the Beijing Key Laboratory of New Drug Mechanisms and Pharmacological Evaluation Study (No BZ0150), the Peking Union Medical College Youth Fund, and the Fundamental Scientific Research Funds for Central Public Institutes (№ 2014RC03).

\section{Author contribution}

Nai-hong CHEN directed the research; Shi-feng CHU conceived the experiments and revised the manuscript; Yan GAO performed the behavioral experiments, immunohistochemistry, immunofluorescence, Western blots, and enzyme assays, as well as prepared the figures, analyzed the results and wrote the manuscript; Jian-ping LI and Cong-yuan XIA assisted with the behavioral experiments and enzyme assays; Zheng MOU performed the Western blot analysis; Zhi-lin WEN prepared the figures; Zhao ZHANG, Jia-qing YAN, Zhen-zhen WANG, Wen-bin HE, Gui-ning WEI, and Xiao-feng GUO revised the manuscript. All authors have read and approved the final paper.

\section{References}

1 Cho IH. Effects of Panax ginseng in neurodegenerative diseases. J Ginseng Res 2012; 36: 342-53.

2 Zhang M, An C, Gao Y, Leak RK, Chen J, Zhang F. Emerging roles of Nrf2 and phase II antioxidant enzymes in neuroprotection. Prog Neurobiol 2013; 100: 30-47.

3 Kumar P, Kalonia H, Kumar A. Huntington's disease: pathogenesis to animal models. Pharmacol Rep 2010; 62: 1-14.

4 Browne SE, Beal MF. Oxidative damage in Huntington's disease pathogenesis. Antioxid Redox Signal 2006; 8: 2061-73.

5 Brouillet E, Hantraye P. Effects of chronic MPTP and 3-nitropropionic acid in nonhuman primates. Curr Opin Neurol 1995; 8: 469-73.

6 Beal MF, Brouillet E, Jenkins BG, Ferrante RJ, Kowall NW, Miller JM, et al. Neurochemical and histologic characterization of striatal excito- toxic lesions produced by the mitochondrial toxin 3-nitropropionic acid. J Neurosci 1993; 13: 4181-92.

7 Wu CL, Hwang CS, Chen SD, Yin JH, Yang DI. Neuroprotective mechanisms of brain-derived neurotrophic factor against 3-nitropropionic acid toxicity: therapeutic implications for Huntington's disease. Ann N Y Acad Sci 2010; 1201: 8-12.

8 Nam E, Lee SM, Koh SE, Joo WS, Maeng S, Im HI, et al. Melatonin protects against neuronal damage induced by 3-nitropropionic acid in rat striatum. Brain Res 2005; 1046: 90-6.

9 Perez-De La Cruz V, Elinos-Calderon D, Robledo-Arratia Y, MedinaCampos ON, Pedraza-Chaverri J, Ali SF, et al. Targeting oxidative/ nitrergic stress ameliorates motor impairment, and attenuates synaptic mitochondrial dysfunction and lipid peroxidation in two models of Huntington's disease. Behav Brain Res 2009; 199: 210-7.

10 Kumar P, Kumar A. Possible role of sertraline against 3-nitropropionic acid induced behavioral, oxidative stress and mitochondrial dysfunctions in rat brain. Prog Neuropsychopharmacol Biol Psychiatry 2009; 33: 100-8.

11 Kumar P, Kumar A. Protective effects of epigallocatechin gallate following 3-nitropropionic acid-induced brain damage: possible nitric oxide mechanisms. Psychopharmacology 2009; 207: 257-70.

12 Garcia E, Limon D, Perez-De La Cruz V, Giordano M, Diaz-Munoz M, Maldonado PD, et al. Lipid peroxidation, mitochondrial dysfunction and neurochemical and behavioural deficits in different neurotoxic models: protective role of S-allylcysteine. Free Radic Res 2008; 42: 892-902.

13 Tunez I, Feijoo M, Collado JA, Medina FJ, Pena J, Munoz Mdel C, et al. Effect of testosterone on oxidative stress and cell damage induced by 3-nitropropionic acid in striatum of ovariectomized rats. Life Sci 2007; 80: 1221-7.

14 Tunez I, Montilla P, del Carmen Munoz M, Medina FJ, Drucker-Colin R. Effect of transcranial magnetic stimulation on oxidative stress induced by 3-nitropropionic acid in cortical synaptosomes. Neurosci Res 2006; 56: 91-5.

15 Yang L, Calingasan NY, Chen J, Ley JJ, Becker DA, Beal MF. A novel azulenyl nitrone antioxidant protects against MPTP and 3-nitropropionic acid neurotoxicities. Exp Neurol 2005; 191: 86-93.

16 Fontaine MAL, Geddes JW, Banks A, Butterfield DA. Effect of exogenous and endogenous antioxidants on 3-nitropropionic acidinduced in vivo oxidative stress and striatal lesions: insights into Huntington's disease. J Neurochem 2000; 75: 1709-15.

17 Cabezas R, El-Bacha RS, Gonzalez J, Barreto GE. Mitochondrial functions in astrocytes: neuroprotective implications from oxidative damage by rotenone. Neurosci Res 2012; 74: 80-90.

18 Peshavariya HM, Dusting GJ, Selemidis S. Analysis of dihydroethidium fluorescence for the detection of intracellular and extracellular superoxide produced by NADPH oxidase. Free Radic Res 2007; 41: 699-712.

19 Joshi G, Johnson JA. The Nrf2-ARE pathway: a valuable therapeutic target for the treatment of neurodegenerative diseases. Recent Pat CNS Drug Discov 2012; 7: 218-29.

20 Jung KA, Kwak MK. The Nrf2 system as a potential target for the development of indirect antioxidants. Molecules 2010; 15: 7266-91.

21 Magesh S, Chen Y, Hu L. Small molecule modulators of Keap1-Nrf2ARE pathway as potential preventive and therapeutic agents. Med Res Rev 2012; 32: 687-726.

22 Patten DA, Germain M, Kelly MA, Slack RS. Reactive oxygen species: stuck in the middle of neurodegeneration. J Alzheimers Dis 2010; 20: S357-67.

23 Tian H, Zhang B, Di J, Jiang G, Chen F, Li H, et al. Keap1: one stone kills three birds Nrf2, IKKbeta and Bcl-2/Bcl-xL. Cancer Lett 2012; 
325: 26-34.

24 Sykiotis GP, Habeos IG, Samuelson AV, Bohmann D. The role of the antioxidant and longevity-promoting Nrf2 pathway in metabolic regulation. Curr Opin Clin Nutr Metab Care 2011; 14: 41-8.

25 Lee JM, Calkins MJ, Chan K, Kan YW, Johnson JA. Identification of the NF-E2-related factor-2-dependent genes conferring protection against oxidative stress in primary cortical astrocytes using oligonucleotide microarray analysis. J Biol Chem 2003; 278: 12029-38.

26 Calabrese V, Cornelius C, Mancuso C, Pennisi G, Calafato S, Bellia F, et al. Cellular stress response: a novel target for chemoprevention and nutritional neuroprotection in aging, neurodegenerative disorders and longevity. Neurochem Res 2008; 33: 2444-71.

27 Criscuolo F, Gonzalez-Barroso Mdel M, Le Maho Y, Ricquier D, Bouillaud F. Avian uncoupling protein expressed in yeast mitochondria prevents endogenous free radical damage. Proc Biol Sci 2005; 272 : 803-10.

28 Jiang D, Chen K, Lu X, Gao HJ, Qin ZH, Lin F. Exercise ameliorates the detrimental effect of chloroquine on skeletal muscles in mice via restoring autophagy flux. Acta Pharmacol Sin 2014; 35: 135-42.

29 Zhang DD. The Nrf2-Keap1-ARE signaling pathway: The regulation and dual function of Nrf2 in cancer. Antioxid Redox Signal 2010; 13 : 1623-6.

30 Fontaine MAL, Geddes JW, Banks A, Butterfield DA. 3-Nitropropionic acid induced in vivo protein oxidation in striatal and cortical synaptosomes: insights into Huntington's disease. Brain Res 2000; 858:
356-62.

31 Kumar P, Padi SSV, Naidu PS, Kumar A. Effect of resveratrol on 3-nitropropionic acid-induced biochemical and behavioural changes: possible neuroprotective mechanisms. Behav Pharmacol 2006; 17: 485-92.

32 Calabrese V, Guagliano E, Sapienza M, Panebianco M, Calafato S, Puleo E, et al. Redox regulation of cellular stress response in aging and neurodegenerative disorders: role of vitagenes. Neurochem Res 2007; 32: 757-73.

33 Calabrese V, Boyd-Kimball D, Scapagnini G, Butterfield DA. Nitric oxide and cellular stress response in brain aging and neurodegenerative disorders: the role of vitagenes. In Vivo 2004; 18: 245-67.

34 Banaclocha MM. Therapeutic potential of $\mathrm{N}$-acetylcysteine in agerelated mitochondrial neurodegenerative diseases. Med Hypotheses 2001; 56: 472-7.

35 Sandhir R, Sood A, Mehrotra A, Kamboj SS. N-Acetylcysteine reverses mitochondrial dysfunctions and behavioral abnormalities in 3-nitropropionic acid-induced Huntington's disease. Neurodegener Dis 2012; 9: 145-57.

36 Calkins MJ, Jakel RJ, Johnson DA, Chan K, Kan YW, Johnson JA. Protection from mitochondrial complex II inhibition in vitro and in vivo by Nrf2-mediated transcription. Proc Natl Acad Sci U S A 2005; 102: 244-9.

37 Linseman DA. Targeting oxidative stress for neuroprotection. Antioxid Redox Signal 2009; 11: 421-4. 